\title{
A unified finite element method for the simulation of hydraulic fracturing with and without fluid lag
}

\author{
J.Q. Bao ${ }^{[a, b]^{*}}$, E. Fathi $^{[\mathrm{ac}]}, \mathrm{S}$. Ameri $^{[\mathrm{b}]}$ \\ ${ }^{a}$ Department of Petroleum and Natural Gas Engineering, West Virginia University, \\ Morgantown, WV 26506, USA \\ ${ }^{\mathrm{b}}$ Research Institute of Petroleum Exploration and Development, PetroChina, Beijing \\ 100083, China
}

\begin{abstract}
Hydraulic fracturing with and without fluid lag have different flow boundary conditions at the fluid front, which always results in different simulation methods. In this paper, we extend a finite element method [1] to simulate hydraulic fracturing with and without fluid lag in a unified manner. A unified numerical boundary condition is imposed on the fluid front independent of fluid lag situations. No effort is needed to track the fluid front explicitly, and the burden of model re-meshing induced by fluid front advancement is avoided. The method is verified by comparing numerical simulations with some analytical solutions. The simulations cover hydraulic fracturing with constant fluid lag fraction, without fluid lag, and with vanishing fluid lag. Some factors governing the simulations are discussed.
\end{abstract}

Keywords: Hydraulic fracturing; fluid lag; finite element method; boundary conditions

\section{Introduction}

Hydraulic fracturing is defined as the process where the propagation of a fracture is driven by the injection of pressurized fluid into the host solid medium [2]. Hydraulic

\section{Nomenclature}

\footnotetext{
*Corresponding author, E-mail address: bjq05@mails.tsinghua.edu.cn.
} 


\begin{tabular}{|c|c|}
\hline $\boldsymbol{B}$ & to transfer pressure into equivalent node force \\
\hline $\boldsymbol{C}$ & equivalent node force of confining stress \\
\hline$D$ & elastic stiffness tensor \\
\hline$e_{l}$ & relative error of half fracture length \\
\hline$e_{p(0)}$ & relative error of fluid pressure at the injection point \\
\hline$e_{w(0)}$ & relative error of fracture width at the injection point \\
\hline$e_{\xi_{f}}$ & relative error of $\xi_{f}$ \\
\hline E & elastic modulus \\
\hline$E^{\prime}$ & modified elastic modulus \\
\hline $\boldsymbol{F}$ & $\begin{array}{l}\text { equivalent global nodal force of the pressure and the confining } \\
\text { stress }\end{array}$ \\
\hline$K_{I}$ & stress intensity factor \\
\hline$K_{I}^{u}$ & upper limit of $K_{I}$ \\
\hline$K_{I C}$ & fracture toughness \\
\hline$K_{m}$ & dimensionless fracture toughness \\
\hline$K^{\prime}$ & modified fracture toughness \\
\hline $\boldsymbol{K}_{u}$ & global stiffness of the solid elements \\
\hline $\boldsymbol{K}_{w}$ & the assembly of the flux stiffness of the fluid elements \\
\hline $\boldsymbol{H}$ & to account for the contribution of fluid boundary conditions \\
\hline$l$ & half fracture length \\
\hline$l_{e}$ & expected half fracture length \\
\hline$l_{N}$ & half fracture length obtained by the numerical method \\
\hline$l_{S}$ & half fracture length in the self-similar solutions \\
\hline $\boldsymbol{L}$ & the assembly of the length stiffness of the fluid elements \\
\hline$n$ & unit outward normal of fracture \\
\hline$p$ & net pressure \\
\hline
\end{tabular}




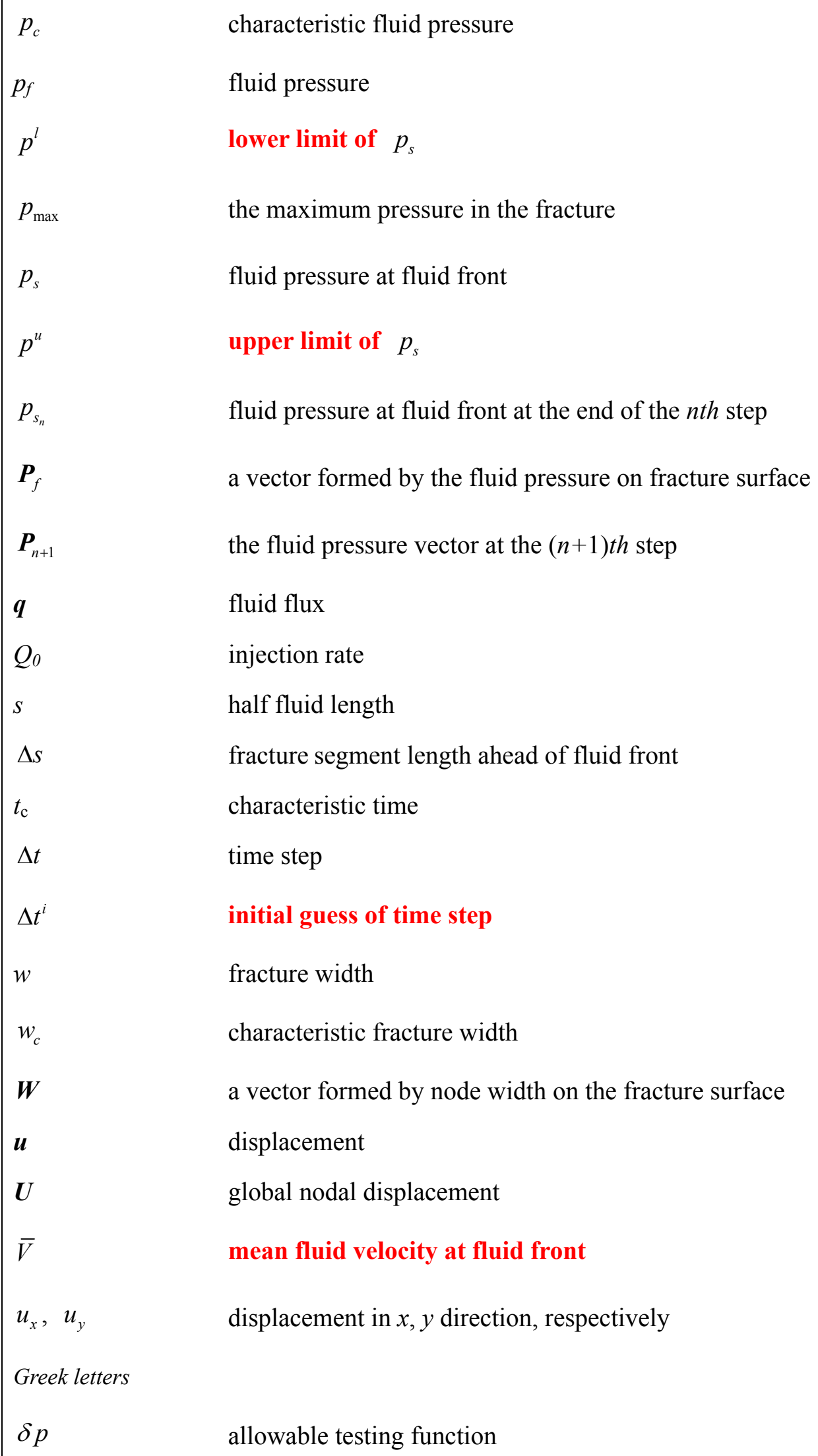




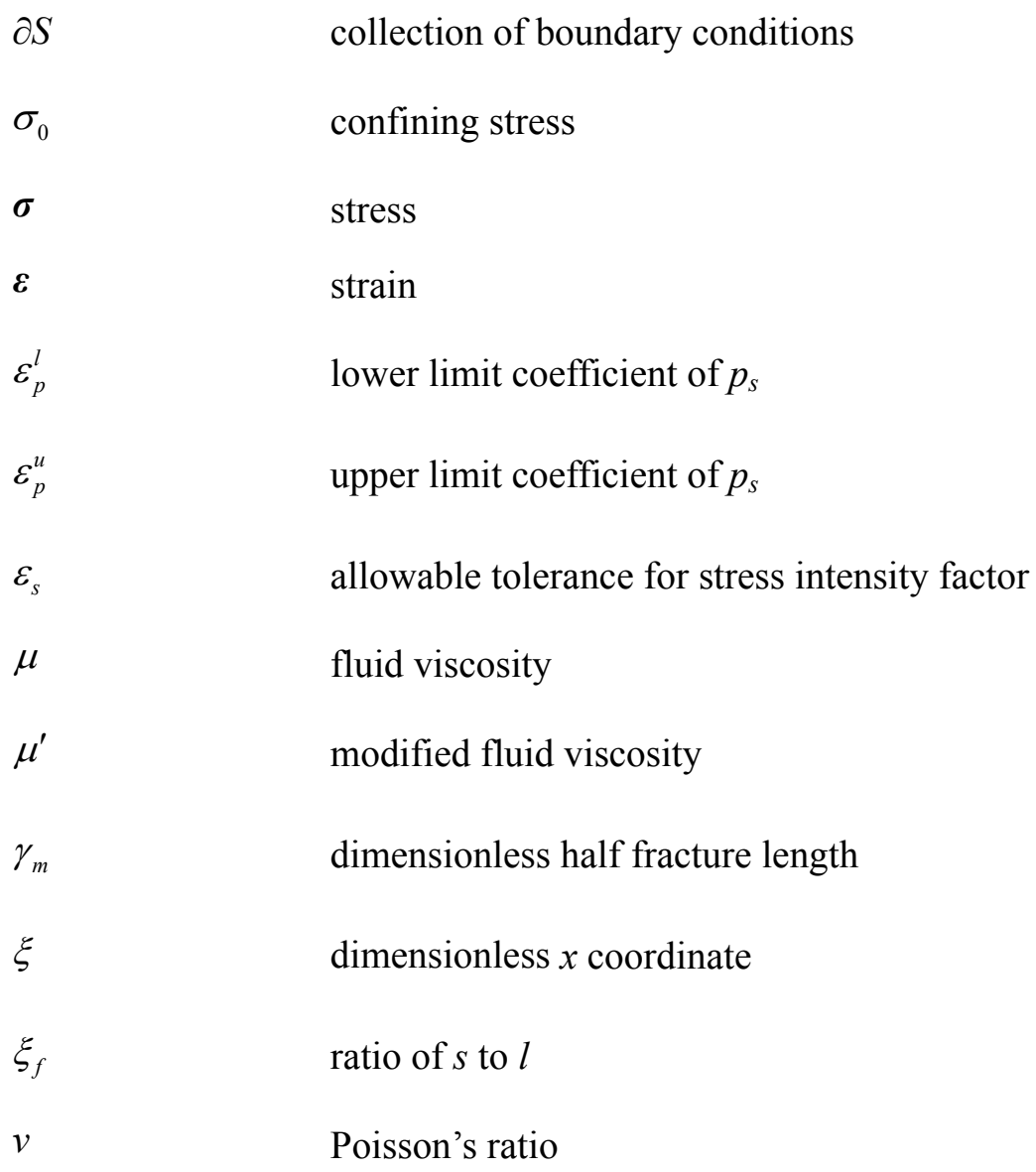

fracturing can be found in natural occurrences such as the formation of the magma-driven dike $[3,4]$, and the growth of fracture along glacier beds driven by water [5]. Actually, hydraulic fracturing has been accepted as an important technique to improve the recovery of conventional and unconventional petroleum and natural gas resources in deep strata, and to remediate waste [6] and induce cave in mining near a free surface [7].

A gap zone between the fluid front and the fracture tip may exist in hydraulic fracturing. This gap zone is referred to as fluid lag. The existence of a fluid lag near the fracture tip has been realized since 1950s [8-10]. In some cases the fluid lag zone is a clear one [11], while in some other cases fluid flow from the solid medium into the fracture is inferred from experiments when the solid medium has high permeability [12]. Fluid lags have huge impact on hydraulic fracturing [13, 
14]. A couple of investigators discussed the factors that govern fluid lags. Garagash [15] discussed the effect of energy dissipation regimes on fluid lags when there is no confining stress. It is found in his discussion that a fluid lag exists and plays an important role when the fracture propagation regime [16] is viscosity-dominated, and it does not exist when the fracture propagation regime is toughness-dominated. Lecampion and Detournay [11] discussed the effect of confining stress on fluid lag evolution, and discovered that a fluid lag tends to vanish when the medium is applied with non-zero confining stress. These investigations imply that in deep strata a fluid lag may gradually vanish following its appearance and extension, and in shallow subsurface a fluid lag may appear and extend even when the fracture is completely filled by the fluid on the beginning. It is desirable to find a unified simulation method that can simulate hydraulic fracturing with and without fluid lag to take these fluid lag situations into account.

Simulation of hydraulic fracturing is notoriously difficult as fracture width is globally dependent on fluid pressure and confining stress, and fluid flow in fractures is non-linearly related to fracture width [11]. Additional difficulties will be encountered if fluid lag situations are accounted for. The additional difficulties arise from two facts. The first one is that efforts are needed to track the fluid front that is always a priori unknown when a fluid lag exists [11, 14]. The second one is that boundary conditions on the fluid front are dependent on fluid lag situations. They are Dirichlet ones when a fluid lag exists; otherwise they are Neumann ones and there is no Dirichlet boundary condition for the fluid zone within the fracture. The changed boundary conditions lead to the proposal of a variety of separate methods to simulate hydraulic fracturing with and without fluid lag.

Garagash [17], Adachi and Detournay [18, 19], Garagash and Detournay[20], Spence and Sharp [21], and $\mathrm{Hu}$ and Garagash [22] simulated hydraulic fracturing without fluid lag based on the displacement discontinuity (DD) method. Chen et al [23], Chen [24], and [25] simulated hydraulic fracturing without fluid lag using a 
cohesive zone model based on the finite element method. Bao et al [1] proposed a coupled finite element method to simulate hydraulic fracturing without fluid lag, accompanied by the introduction of a condensation technique to accelerate its simulation [26]. Zhang [14] et al proposed a DD method to simulate hydraulic fracturing with a fluid lag, followed by Gordeliy and Detournay [27] using an updated scheme to search fluid front position. Hunsweck et al [28] developed a finite element method to simulate hydraulic fracturing with a fluid lag. Gordeliy and Peirce [29] proposed a $P$ to $W$ extended finite method (XFEM) to simulate hydraulic fracturing with a fluid lag, and a $P \& W$ one to simulate hydraulic fracturing without fluid lag. Although Shen [30] introduced a DD method that can simulate hydraulic fracturing with and without fluid lag uniformly, some improvement measures are needed in the method when fracture propagation regimes are toughness-dominated.

In this paper we extend the coupled method in [1] to simulate hydraulic fracturing with and without fluid lag in a unified way. The unified method is proposed based on the application of a unified boundary condition on the fluid front, regardless of fluid lag situations. No effort is needed to track fluid front when a fluid lag exists, meaning that the burden of model remeshing induced by a fluid lag [28] is avoided. For simplicity, we present the unified finite element method and its simulations in a symmetric plane strain model, and use an element-wise technique [31] to remesh the model when fracture propagates. We assume that the confining stress has a constant orientation. This assumption indicates that fractures propagate along a straight line that is perpendicular to the confining stress. It is worth pointing out that the unified finite element method is independent of model remeshing techniques and the confining stress assumption, and it can be implanted into XFEM and the generalized finite element method [32]. Like in $[14,27,28,30]$ we ignore bubble pressure in the fluid lag zone in our method. In the article we focus our method on the simulation of hydraulic fracturing without leak-off, extension of the method to simulate hydraulic fracturing with leak-off is straightforward if the fluid lag zone is a clear one. 
The rest of this paper is organized as follows: the theoretical model of hydraulic fracturing without leak-off is presented in section 2. The unified method is introduced in section 3. The verification of the unified finite element method is presented in section 4, as well as discussions. Conclusions are made in section 5.

\section{Theoretical model}

In the theoretical model, hydraulic fracturing is caused by the injection of incompressible Newtonian fluid into a solid medium. There are two possibilities during the process of hydraulic fracturing. For the first possibility, the fluid completely fills the fracture as shown in Fig. 1(a). For the second possibility, there is a fluid lag between the fluid front and the fracture tip as shown in Fig. 1(b). Let $Q_{0}$ denote the injection rate. Due to the symmetry of the fracture, half of the injected fluid flows into the half fracture as shown in Fig. 1. Fracture width $w$ as shown in Fig. 1 results from the action of the far field confining stress $\sigma_{0}$ and the fluid pressure applied on the fracture surface. The injection point is taken as the origin of the Cartesian coordinate used in this paper, and the fracture propagates in $x$ direction. The theoretical model includes the elastic response of the solid medium and the fluid flow in the fracture. 


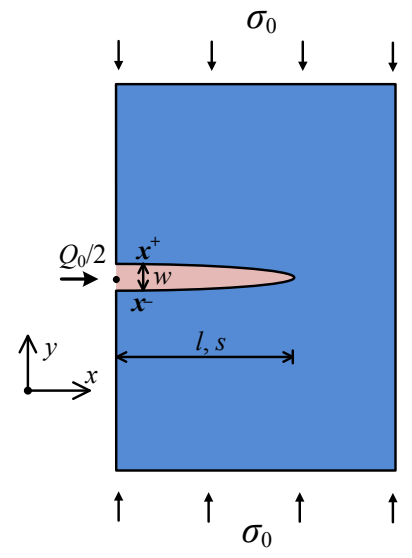

(a)

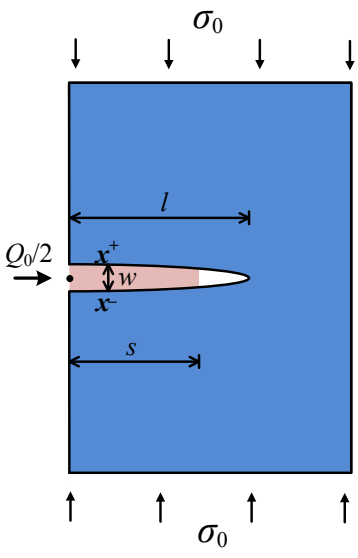

(b)

- Injection point $\square$ Injected fluid $\square$ Solid medium

Fig. 1. Hydraulic fractures in half space: (a) without fluid lag; (b) with a fluid lag.

\section{(i) Elastic response}

The elastic response at any point $\boldsymbol{x}$ in the solid medium is governed by the constitutive law and the equivalence condition, of which the equations are:

$$
\sigma(\boldsymbol{x})=\boldsymbol{D}: \varepsilon(\boldsymbol{x})
$$

and

$$
\nabla \cdot \boldsymbol{\sigma}=0
$$

In Eq. (1) and (2), $\boldsymbol{\sigma}$ is the stress tensor, $\boldsymbol{D}$ is the elastic stiffness tensor of the solid medium, $(\nabla \cdot)$ is the divergence operator, $(:)$ is the double dot product operator between two tensors, and $\varepsilon$ is the strain tensor and equals the symmetric part of the gradient of displacement $\boldsymbol{u}$, i.e.

$$
\boldsymbol{\varepsilon}=\left[\nabla \boldsymbol{u}+(\nabla \boldsymbol{u})^{T}\right] / 2,
$$

In Eq. (3), ( $\nabla)$ is the gradient operator, and superscript $T$ stands for transpose.

The stress boundary conditions can be expressed as

(i) on the fracture surface

$$
\boldsymbol{\sigma}(x, 0) \cdot \boldsymbol{n}=-p_{f}(x) \boldsymbol{n} x \leq s
$$


(ii) on the far field as shown in Fig. 1

$$
\boldsymbol{\sigma} \cdot \boldsymbol{n}=-\sigma_{0} \boldsymbol{n}
$$

where $p_{f}$ is the fluid pressure in the fracture, $\boldsymbol{n}$ is the outward unit normal, and $s$ as shown in Fig. 1 is the half fluid length.

Due to symmetry, the displacement boundary condition in Fig. 1 can be expressed as

$$
u_{x}(0, y)=0
$$

where $u_{x}$ is the displacement in $x$ direction.

In hydraulic fracturing, fracture propagation is dominated by the normal opening mode, and the propagation criterion is

$$
K_{I}=K_{I C}
$$

where $K_{I}$ is the stress intensity factor for the tensile opening mode of the fracture, and $K_{I C}$ is the fracture toughness of the solid medium.

\section{(ii) Fluid flow in the fracture}

As shown in Fig. 1, the fracture width $w$ is expressed as

$$
w(\boldsymbol{x})=\left[u_{y}\left(\boldsymbol{x}^{+}\right)-u_{y}\left(\boldsymbol{x}^{-}\right)\right],
$$

where $u_{y}$ is the displacement in $y$ direction, and $\boldsymbol{x}^{+}$and $\boldsymbol{x}^{-}$are two points on the fracture surface as shown in Fig. 1. These two points are actually the same point $\boldsymbol{x}(x$, $0)$ before fracture tip reaches there.

The one-dimensional fluid flow in the fracture is modeled with the lubrication theory, and its equation is described by Poiseuille's law [33] 


$$
\boldsymbol{q}=-\frac{w^{3}}{12 \mu} \nabla p_{f}
$$

where $\boldsymbol{q}$ is the fluid flux, $\mu$ is the fluid viscosity, and $(\nabla)$ is the gradient operator defined in $x$ direction in Fig. 1. By ignoring the compressibility of the injected fluid, the conservation of the fluid in the fracture leads to [34]

$$
\frac{\partial w}{\partial t}+\nabla \cdot \boldsymbol{q}=0
$$

where $(\nabla \cdot)$ is the divergence operator defined in $x$ direction in Fig. 1.

The boundary condition at the injection point is cast as

$$
\boldsymbol{q}(0)=Q_{0} / 2
$$

The boundary condition on the fluid front is dependent on fluid lag situations. If there is no fluid lag in the fracture, the boundary condition is a Neumann one and it is written as

$$
\boldsymbol{q}(l)=0
$$

where $l$ as shown in Fig. 1 is the half fracture length. However, if a fluid lag exists, the boundary condition is a Dirichlet one, and it is written as

$$
p_{f}(s)=0
$$

where $s$ is the half fluid length as shown in Fig. 1(b) and it is a priori unknown. As the boundary condition at the fluid front is dependent on the fluid lag situation, it will switch from the Neumann one to the Dirichlet one if a fluid lag appears, and vise versa if a fluid vanishes. The different boundary conditions in Eqs. (12) and (13) always lead to different simulation method. In addition, if there is a fluid lag in

the fracture, the pace of fluid front advancement is different from that of fracture propagation, otherwise their paces are the same.

\section{Unified finite element method}




\section{(i) Unified boundary condition}

The different boundary conditions in Eqs. (12-13) are the origin that lead to different simulation methods. The underlying assumption of Eq. (13) is that no suction zone exists over the whole period of hydraulic fracturing and the fluid front moves continuously. As shown in Fig. 2, a suction zone is defined a zone where fluid pressure is below than zero. A suction zone is allowed to exist in our numerical method. The fluid front does not move until the suction zone vanishes, followed by the appearance of a new suction zone. Based on this assumption, we can use a unified Neumann boundary condition at the fluid front regardless of fluid lag situations, and this condition is expressed as

$$
\boldsymbol{q}(s)=0
$$

Similarly, a unified Dirichlet-like boundary condition is used in [30].

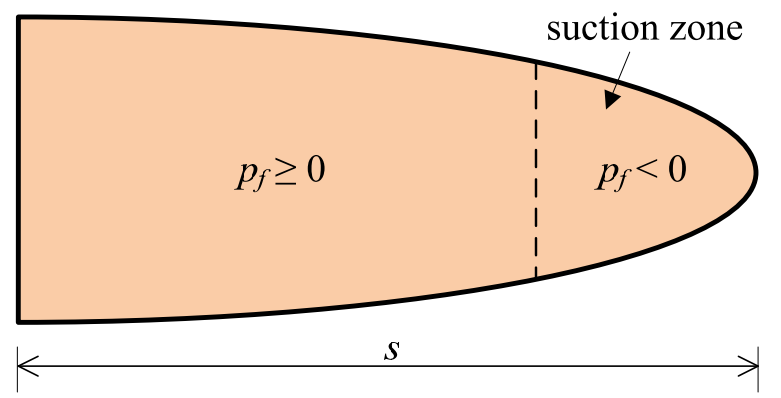

Fig. 2. Fluid regime with a suction zone in a hydraulic fracture.

\section{(ii) Unified finite element method}

With the unified boundary condition in Eq. (14), we extend the finite element method in [1] to simulate hydraulic fracturing with and without fluid lag. The finite element equations are from the $2 \mathrm{D}$ elastic response of the solid medium and the $1 \mathrm{D}$ fluid flow within the fracture.

The finite element equation for the elastic response is cast as 


$$
\boldsymbol{K}_{u} \boldsymbol{U}=\boldsymbol{B P}_{f}+\boldsymbol{F}
$$

where $K_{u}$ is the global stiffness of the solid elements, $U$ is the global nodal displacement, $\boldsymbol{P}_{f}$ is a vector formed by the fluid pressure on the fracture surface, matrix $B$ plays the role to transfer the pressure into equivalent node force, and $F$ is the equivalent global nodal force of the confining stress.

Using the condensation technique in [26], from Eq. (15) we have

$$
\boldsymbol{W}=\boldsymbol{K} \boldsymbol{P}_{f}+\boldsymbol{C}
$$

where $W$ is a vector formed by the width of the nodes on the fracture surface, $K$ is a matrix that relates fracture width to fluid pressure, $C$ is the fracture closure induced by the confining stress.

The 1D finite elements in the fluid domain are set up by fracture segments. Eq. (10) yields its weak form as [35]

$$
\int_{0}^{s}\left[-\nabla(\delta p) \cdot \boldsymbol{q}+(\delta p) \frac{\partial w}{\partial t}\right] d l+\left.\delta p q\right|_{\partial S}=0
$$

where $\delta p$ is any allowable testing function, and $\partial S$ is the collection of boundary conditions in Eqs. (11) and (15). Therefore, a finite element equation for fluid flow in the fracture is cast as

$$
\boldsymbol{K}_{w}(\boldsymbol{W}) \boldsymbol{P}_{f}+\boldsymbol{L} \dot{\boldsymbol{W}}+\boldsymbol{H}=\mathbf{0}
$$

where $\boldsymbol{W}$ is a vector formed by the width of the nodes on the fracture surface, $\boldsymbol{K}_{\boldsymbol{w}}$ is the assembly of the flux stiffness of the fluid elements and it is a function of $\boldsymbol{W}, \boldsymbol{L}$ is the assembly of the length stiffness of the fluid elements, and $\boldsymbol{H}$ accounts for the contribution of the fluid boundary conditions.

Taken time integration, Eq. (19) yields

$$
\int_{t_{n}}^{t_{n+1}}\left[\boldsymbol{K}_{w}(\boldsymbol{W}) \boldsymbol{P}_{f}+\boldsymbol{L} \dot{\boldsymbol{W}}+\boldsymbol{H}\right] d t=\mathbf{0}
$$


Backward Euler scheme for time difference is used in this paper. So according to Eqs. (19), we have

$$
\boldsymbol{K}_{w}\left(\boldsymbol{W}_{n+1}\right) \boldsymbol{P}_{n+1} \Delta t+\boldsymbol{L}\left(\boldsymbol{W}_{n+1}-\boldsymbol{W}_{n}\right)+\boldsymbol{H} \Delta t=\boldsymbol{O}
$$

where $\boldsymbol{P}_{n+1}$ and $\boldsymbol{W}_{n+1}$ are the fluid pressure and fracture width vectors in the fluid-filled fracture zone at the $(n+1) t h$ step, respectively, and $P_{n}$ and $W_{n}$ are their corresponding ones at the nth step, and $\Delta t$ is the time step.

The unified finite element method obtains $\boldsymbol{P}_{n+1}$ and $\boldsymbol{W}_{n+1}$ by solving Eq. (20) and

$$
\boldsymbol{W}_{n+1}=\boldsymbol{E} \boldsymbol{P}_{n+1}+\boldsymbol{C}
$$

regardless of fluid lag situations , where Eq. (21) is originated from Eq. (17). It is seen in Eqs. (20-21) that their sizes are dependent on the number of nodes in the fluid zone. The sizes will increase when the fluid front moves forward in our method, as elements behinds the fracture tip are not adjusted during the simulations.

(iii) Numerical schemes

It is a heavy burden for a finite element method to seek solutions where both Eq. (7) and Eq. (13) are extremely approximated when fluid lag exists. The heavy burden arises from tracking the fluid front $[14,27]$ and the fracture tip, and from model remeshing to keep elements conform to the fluid front and the fracture tip. We seek physically-acceptable solutions rather than extremely-closed ones in our method to circumvent the simulation burden induced by a fluid lag.

For the physically-acceptable solutions in a step, they are required to satisfy 


$$
\begin{gathered}
p_{s} \geq p^{l} \\
p_{s}<p^{u} \text { if } s<l \\
K_{I}<K_{I C} \text { if } s<l \\
K_{I C} \leq K_{I} \leq K_{I}^{u} \text { if } s=l
\end{gathered}
$$

where $p_{s}$ is the fluid pressure at the fluid front, $p^{l}$ is its lower limit, $p^{u}$ is its upper limit when a fluid lag exists, and $K_{I}^{u}$ is the upper limit of the stress intensity factor when no fluid lag exists. Eq. (13) is approximated by Eqs. (22-1, 2) when a fluid lag exist, while meanwhile Eq. (7) is relaxed to a large extent. Eq. (7) is approximated by Eq. (22-4) when there is no fluid lag.

The conditions in Eq. (22) are guaranteed with a trial and error procedure by changing $\Delta t$ while keeping $s$ fixed in a step. The strategy of the procedure depends on the fluid lag situations in a trial step.

If a fluid lag exists at a trial step, we first find $\Delta t$ that enables the trial solutions to satisfy the first two conditions in Eq. (22). If the third condition in Eq. (22) is not satisfied, it indicates that the fracture has already propagated before the suction zone vanishes in the step. In this case we change $l$ to update the fracture tip and repeat the trial until all the first three conditions in Eq. (22) are satisfied.

If no fluid lag exists at a trial step, we first find $\Delta t$ that enables the trial solutions to satisfy the last condition in Eq. (22). If the first condition in Eq. (22) is not satisfied, it means that the fracture has already propagated before the suction zone vanishes in the step. This will yield a fluid lag in the step. In this case we also change $l$ to update the fracture tip and restart the trial step considering a fluid flag. Otherwise the simulation will move into a new step. 
The flowchart of a step is shown in Fig. 3.

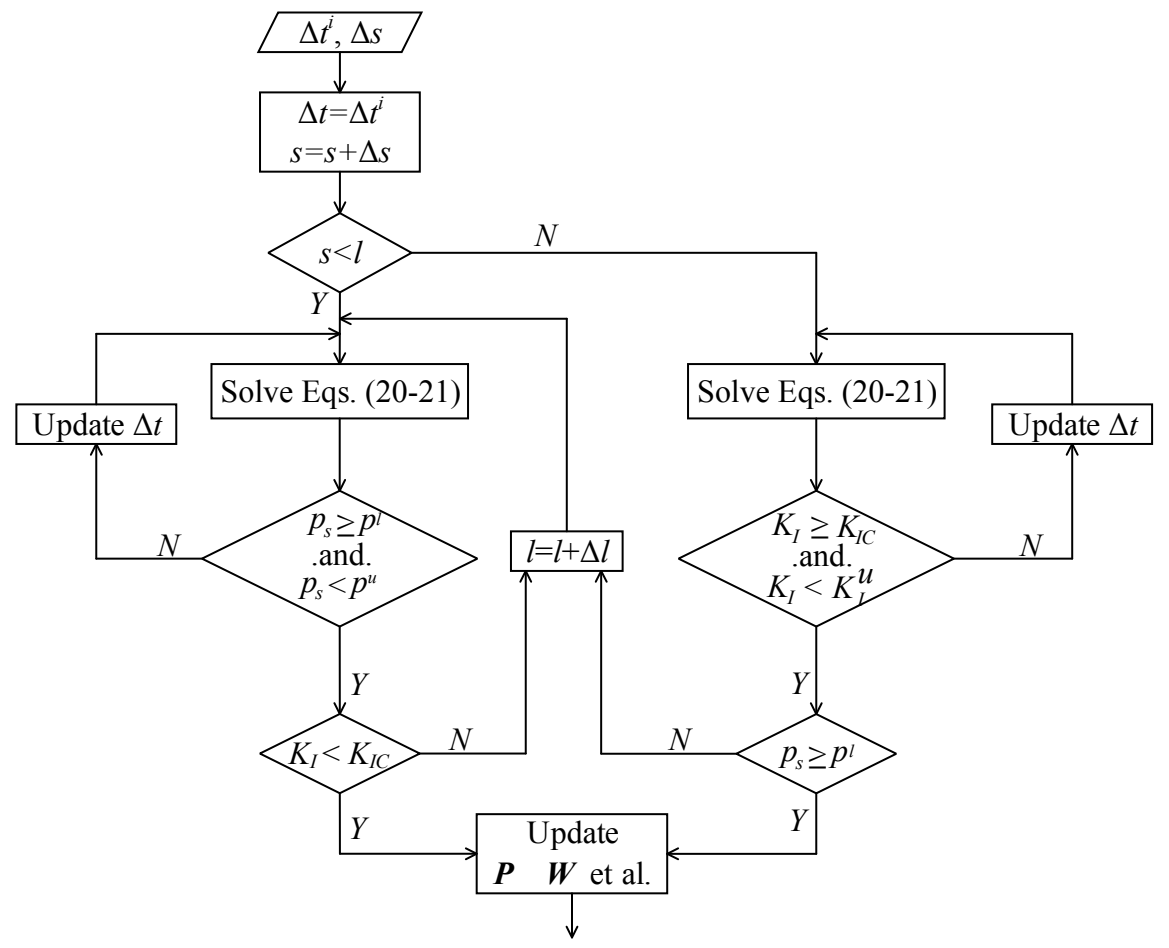

Fig. 3. Flowchart of a step in the numerical method.

In Fig. $3 \Delta t$ is the initial guess of $\Delta t, \Delta s$ is the fluid front movement size in the step, and $\Delta l$ is the propagation size. We use the element-size technique in $[1,26]$ to determine $\Delta l$ when fractures propagates and $\Delta s$ when there is no suction zone in the last step. When the suction zone does not vanish in the last step, $\Delta s$ is set to be zero in the trial step. It is seen in Fig. 8 that the fracture tip may change a couple of times if the fracture propagates much faster than the fluid front advances. When the suction zone vanishes in the last step, $\Delta t^{i}$ is determined by

$$
\Delta t^{i}=\Delta s / \bar{V}
$$

where $\bar{V}$ is the mean fluid velocity at the fluid front [28]; otherwise the time step in last step is taken as $\Delta t^{i}$.

The upper limit of stress intensity factor is

$$
K_{I}^{u}=\left(1.0+\varepsilon_{s}\right) K_{I C}
$$


where $\varepsilon_{s}$ is the allowable tolerance for stress intensity factor. The lower limit of $p_{s}$ is

$$
p^{l}=\varepsilon_{p}^{l} p_{\max }
$$

where $\varepsilon_{p}^{l}$ is the lower limit coefficient of $p_{s}$ and $p_{\max }$ is the maximum pressure in the trial step. Similarly, we have

$$
p^{u}=\varepsilon_{p}^{u} p_{\max }
$$

where $\varepsilon_{p}^{u}$ is the upper limit coefficient of $p_{s}$.

Fluid front advancement and fracture propagation are two processes that interact with each other when a fluid lag exists. Tt is a tough task for a finite element method to find a set of $\Delta t, \Delta s$ and $\Delta l$ with which both Eq. (7) and Eq. (13) are approximated in great precision. We relax Eq. (7) to some extent when a fluid lag exists. This physically-acceptable relaxation enables us to use an element-wise technique and avoid the simulation burden induced by the fluid lag. The time step is adjusted during the trial and error procedure, indicating that an implicit scheme is used to estimate fluid front advancement velocity when the suction zone vanishes and the fluid lag still exists.

\section{Verification \& discussion}

\section{(i) Model definition}

For hydraulic fracturing in a homogeneous plane strain model, it behaves in a self-similar manner when the injection is constant and there is no confining stress [15]. This self-similar behavior is also observed when there is uniform confining stress and hydraulic fracturing steps into the late-time stage [11]. These self-similar behaviors are dependent on the fracturing propagation regimes, which can be categorized 
according to the dimensionless fracture toughness $K_{m}[16] . K_{m}$ is defined as

$$
K_{m}=\frac{K^{\prime}}{E^{\prime}}\left(\frac{E^{\prime}}{\mu^{\prime} Q_{0}}\right)^{1 / 4}
$$

where

$$
K^{\prime}=4\left(\frac{2}{\pi}\right)^{1 / 2} K_{I C}, \quad E^{\prime}=\frac{E}{1-v^{2}}, \quad \mu^{\prime}=12 \mu
$$

In Eq. (28) $E$ is the elastic modulus and $v$ is the Poisson's ratio. The fracture propagation regime is viscosity-dominated if $K_{m}$ is smaller than 1.0, it is toughness-dominated if $K_{m}$ is greater than 4.0, and otherwise it is intermediate [22].

We verify our numerical method by comparing its numerical results with some self-similar solutions. For simplicity, characteristic variants for time $\left(t_{\mathrm{c}}\right)$, pressure $\left(p_{c}\right)$, and fracture opening $\left(w_{c}\right)$ are defined as

$$
t_{c}=\left[\frac{l_{e}\left(\mu^{\prime}\right)^{1 / 6}}{\left(E^{\prime}\right)^{1 / 6} Q_{0}^{1 / 2} \gamma_{m}}\right]^{3 / 2}, p_{c}=\frac{\left(\mu^{\prime}\right)^{1 / 3}\left(E^{\prime}\right)^{2 / 3}}{t_{c}^{1 / 3}}, w_{c}=\frac{\left(\mu^{\prime}\right)^{1 / 6} Q_{0}^{1 / 2} t_{c}^{1 / 3}}{E^{1 / 6}}
$$

where $l_{e}$ is the expected half fracture length, and $\gamma_{m}$ is defined as the dimensionless half fracture length and it is a function of $K_{m}[15,22] . l_{e}$ equals $13.5 \mathrm{~m}$ in the following simulations. The characteristic variants are used to normalize their corresponding solutions in the simulations.

In the self-similar solutions, $t_{c}$ means the time when $l$ reaches $l_{e}$. We define $e_{l}$ as the relative error of half fracture length, and it is

$$
e_{l}=\left|l_{N}-l_{S}\right| / l_{S}
$$

where $l_{N}$ and $l_{S}$ are half fracture length obtained by the numerical method and self-similar solutions at the same time, respectively. Other relative errors can also be defined in a similar way. 
Net pressure $p$ is defined as

$$
p(\xi)=p_{f}(\xi)-\sigma_{0}(\xi), x \in\left[0, \xi_{f}\right]
$$

where $\xi$ is defined as dimensionless $x$ coordinate and it equals zero at the injection point and equals 1 at the fracture tip, and $\xi_{f}$ is defined as the ratio of $s$ to $l$.

The rectangle model $\mathrm{ABCD}$ shown in Fig. 3 is used to represent an infinite solid medium. The fracture is horizontal and located in the middle left of the model. Point $\mathrm{E}$ in Fig. 3, i.e. the middle of edge $\mathrm{BC}$, is fixed in $y$ direction. Edge $\mathrm{AD}$ is fixed in $x$ direction.

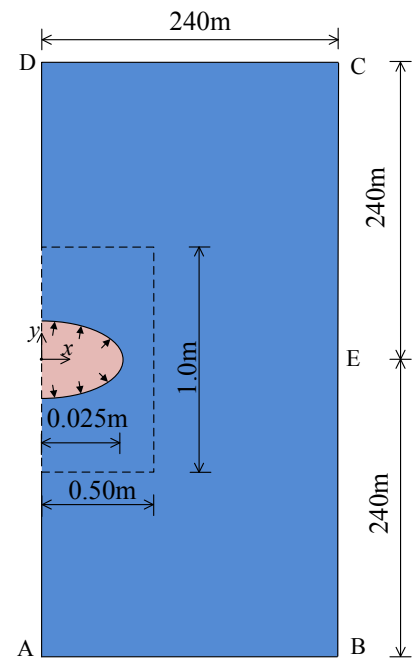

Fig. 3. Representative model (not scaled).

The initial uniform pressure in the fracture in each simulation equals the confining stress plus $0.1 \mathrm{MPa}$. The common parameters for the simulations are listed in Table 1 .

Table 1. Common parameters for the simulations

\begin{tabular}{|c|c|c|}
\hline Elastic modulus & $E$ & $18,000 \mathrm{MPa}$ \\
\hline Poisson's ratio & $v$ & 0.2 \\
\hline Injection rate & $Q_{0}$ & $0.001 \mathrm{~m}^{2} / \mathrm{s}$ \\
\hline
\end{tabular}




\begin{tabular}{l|l|l} 
Dynamic viscosity & $\mu$ & 7.98e-4Pa's
\end{tabular}

\section{(ii) Verification}

Here $\varepsilon_{p}^{u}$ and $\varepsilon_{s}$ equal 0.001 , and $\varepsilon_{p}^{l}$ equals -0.001 . For the elements on the fracture propagation path, their characteristic sizes are $0.025 \mathrm{~m}$. For the elements far away from the fracture zone, the characteristic sizes are $2.0 \mathrm{~m}$. The initial half fracture length is $0.025 \mathrm{~m}$. There are 50632 elements and 51001 nodes in the initial model. The finite elements in the dashed zone in Fig. 4 are shown in Fig. 5.

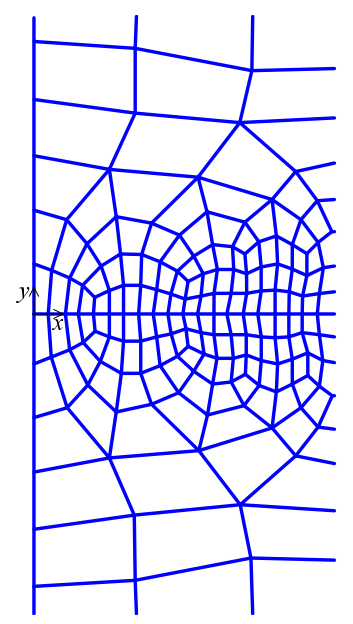

Fig. 5. Finite elements around the injection point.

(1) Simulations with $K_{m}<1, \sigma_{0}=0$

Garagash [15] developed a self-similar solution for hydraulic fracturing with zero far field confining stress. In the self-similar solution, $\xi_{f}$ is a constant and it is dependent on $K_{m}$. The numerical result and self-similar solution of $\xi_{f}$, the normalized half fracture length, and the normalized pressure and the normalized fracture width at the injection point for the case where $K_{m}$ equals 0.4 are plotted in Fig. 5. Here $\gamma_{m}$ equals 1.4919 [28]. 


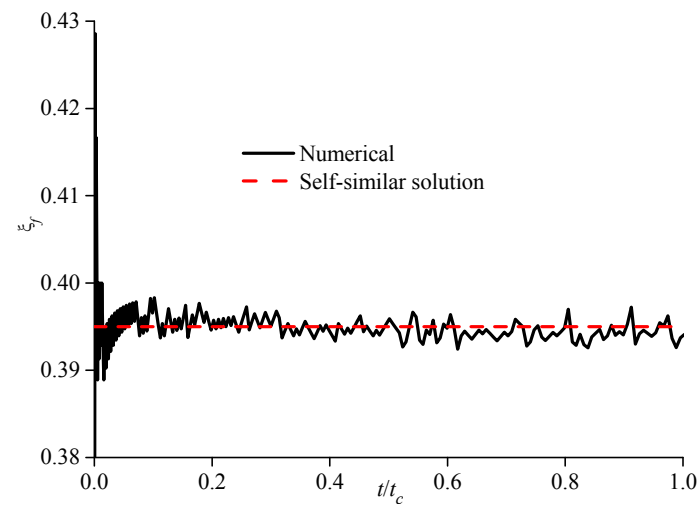

(a)

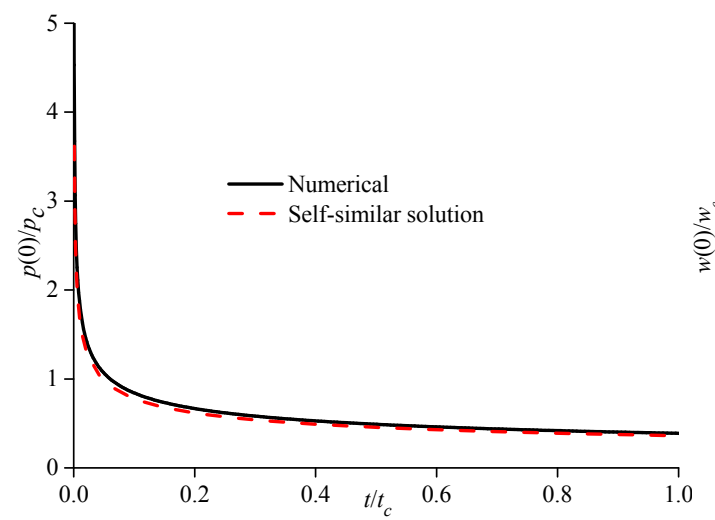

(c)

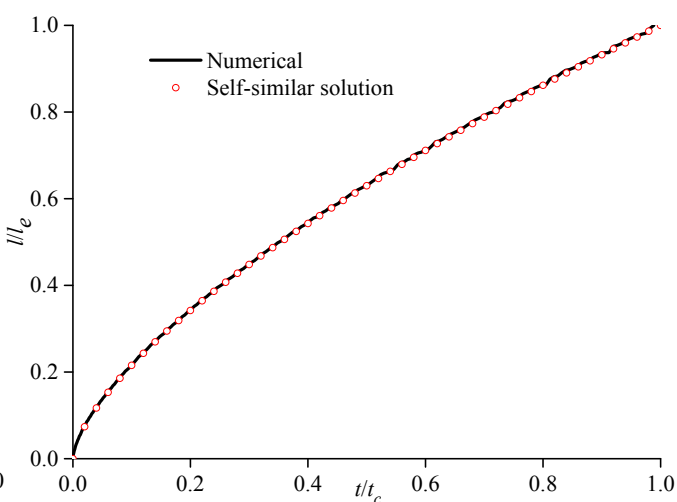

(b)

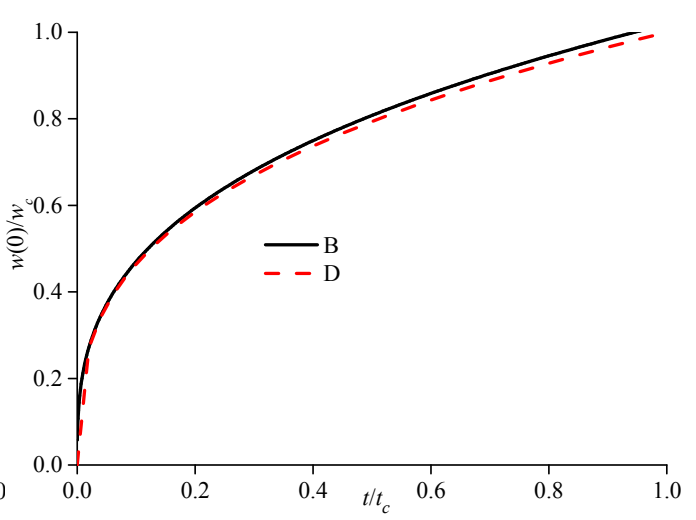

(d)

Fig. 6. Numerical and self-similar solutions of $\xi_{f}$ (a), the normalized half fracture length (b), the normalized pressure at the injection point (c), and the normalized fracture width at the injection point $(\mathrm{d})$.

It is seen in Fig. 6(a) that a fluid lag appears when the fluid is injected into the fracture. It also is seen in Fig. 6(a) that in the numerical solution $\xi_{f}$ has its largest relative errors on the beginning due to the fact that there is one hydraulic element in the initial model. $\xi_{f}$ fluctuates along and is always close to the self-similar solution when the fracture propagates. The relative error of $\xi_{f}$ is smaller than 0.01 when $l$ reaches $l_{e}$. It is seen in Fig. 6(b) - (d) that the numerical results match very well the self-similar solutions. 
A variety of simulations with different $K_{m}$ s were performed with the proposed method. The solutions of $\xi_{f}, p(0)$, and $w(0)$ in each simulation, as well as their relative errors, are listed in Table 2 when $l$ reaches $l_{e}$. The relative errors in Table 2 show that the numerical results have excellent agreement with the self-similar solutions.

Table 2. $\xi_{f}, p(0)$, and $w(0)$ when $l$ reach $l_{e}$, and their relative errors.

\begin{tabular}{|c|c|c|c|c|c|c|c|c|c|c|}
\hline \multirow{2}{*}{$K_{m}$} & \multirow{2}{*}{$t / t_{c}$} & \multicolumn{2}{|c|}{$\xi_{f}$} & \multirow{2}{*}{$e_{\xi_{f}}$} & \multicolumn{2}{|c|}{$p(0)(\mathrm{MPa})$} & \multirow{2}{*}{$e_{p(0)}$} & \multicolumn{2}{|c|}{$w(0)(\mathrm{mm})$} & \multirow{2}{*}{$e_{w(0)}$} \\
\hline & & Exact $^{[28]}$ & Numerical & & Exact $^{[28]}$ & Numerical & & Exact $^{[28]}$ & Numerical & \\
\hline 0.4 & 0.989 & 0.3950 & 0.3926 & 0.006 & 0.3617 & 0.3649 & 0.009 & 0.4644 & 0.4590 & 0.012 \\
\hline 0.5 & 0.991 & 0.5025 & 0.5000 & 0.005 & 0.3436 & 0.3453 & 0.005 & 0.5220 & 0.5179 & 0.008 \\
\hline 0.6 & 0.994 & 0.5997 & 0.5981 & 0.003 & 0.3322 & 0.3334 & 0.004 & 0.5719 & 0.5685 & 0.006 \\
\hline 0.7 & 0.995 & 0.6849 & 0.6833 & 0.002 & 0.3256 & 0.3265 & 0.003 & 0.6150 & 0.6119 & 0.005 \\
\hline 0.8 & 0.995 & 0.7574 & 0.7556 & 0.002 & 0.3224 & 0.3228 & 0.001 & 0.6523 & 0.698 & 0.004 \\
\hline
\end{tabular}

(2) Simulation with $K_{m}=2, \sigma_{0}=0$

It is seen in Table. 2 that $\xi_{f}$ increases with increasing $K_{m}$. Garagash [15] showed that the fluid lag is ignorable when $K_{m}$ is larger than 1.6. We performed a simulation 
with $K_{m}$ equal to 2 and $\sigma_{0}$ equal to 0 , where $\gamma_{m}$ equals 0.5144 [22]. It is found in the simulation that the fluid front always advances when the fracture propagates, and there is no fluid lag all over the simulation. We compared the numerical result with a self-similar solution without fluid lag [22]. Some comparisons are shown in Fig. 7.

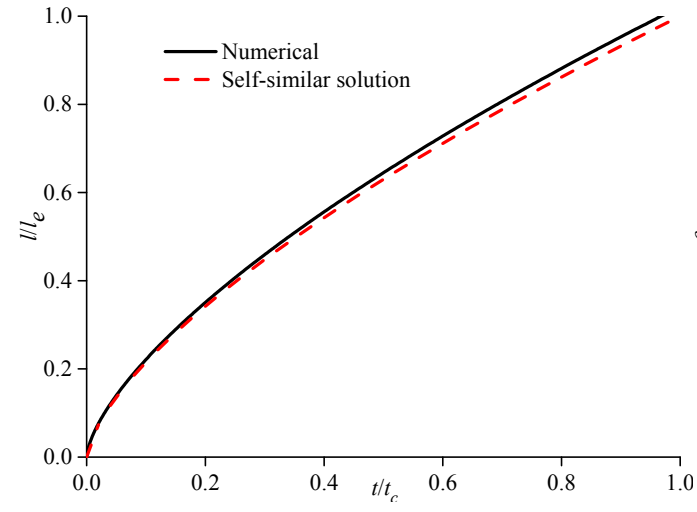

(a)

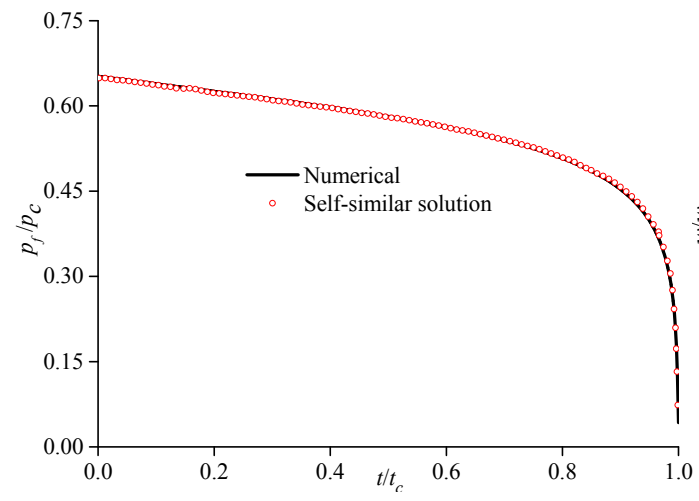

(c)

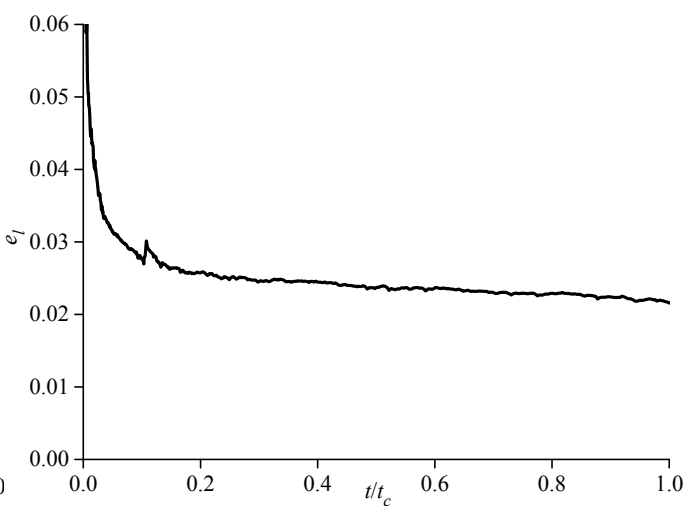

(b)

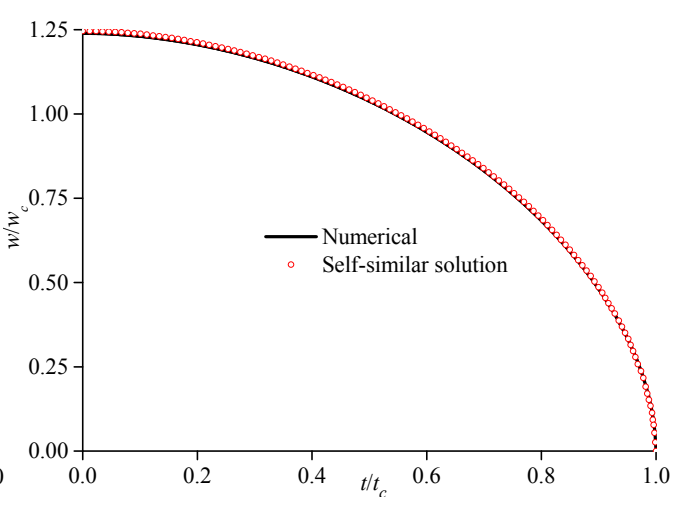

(d)

Fig. 7. Half fracture length (a), $e_{l}(\mathrm{~b})$, the normalized pressure profile (c), and the normalized fracture width profile with $l=13.5 \mathrm{~m}(\mathrm{~d})$.

It is seen in Fig. 7(a) that for the half fracture length, the gap between the numerical result and self-similar solution increases when fracture propagates. However, Fig. 7(b) shows that their relative error generally decreases when the fracture propagates. For the normalized net pressure profiles when $l$ reaches $13.5 \mathrm{~m}$ (see Fig. 6(c)), the numerical result is very close to the self-similar solution. Similar phenomenon is observed for the normalized width profile that is shown in Fig. 6(d). 
(3) Simulation with $K_{m}=0.6, \sigma_{0} \neq 0$

Lecampion [11] showed that the fluid tends to fill the whole fracture when there exists a constant confining stress, and hydraulic fracturing can be described by a self-similar solution without fluid lag in the late time. This description is valid when $t$ is around the order of $\mu^{\prime} E^{\prime 2} / \sigma_{0}^{3}[11]$. We performed a simulation with non-zero constant confining stress, and compared the numerical result with a late-time self-similar solution [20]. In the simulation, $K_{m}$ equals 0.6 , and $\sigma_{0}$ equals $1.5 p_{c}, \gamma_{m}$ equals 0.6148 , and $t_{c}$ equals $15.47 \mathrm{~s}$. Here $\mu^{\prime} E^{\prime 2} / \sigma_{0}^{3}$ equals $4.58 \mathrm{~s}$. Obviously, the simulation is expected to be close to the self-similar solution when $t$ is close to $t_{c}$.

In the numerical method $l$ reaches $l_{c}$ at the moment of $15.014 \mathrm{~s}$, and its evolution of $\xi_{f}$ is plotted in Fig. 8. It is seen in Fig. 8 that on the very beginning the fluid front has already been separated from the fracture tip, and a fluid lag always exists. $\xi_{f}$ gets closer to 1.0 when the fracture propagates. We found $\xi_{f}$ equals 0.996 when $t / t_{c}$ equals 1.0 . Therefore the fluid lag is negligible in the numerical solution when $t$ reaches $t_{c}$, meaning that the numerical results have the fundamental qualification of being close to the self-similar solution [11] when $t$ reaches $t_{\mathrm{c}}$.

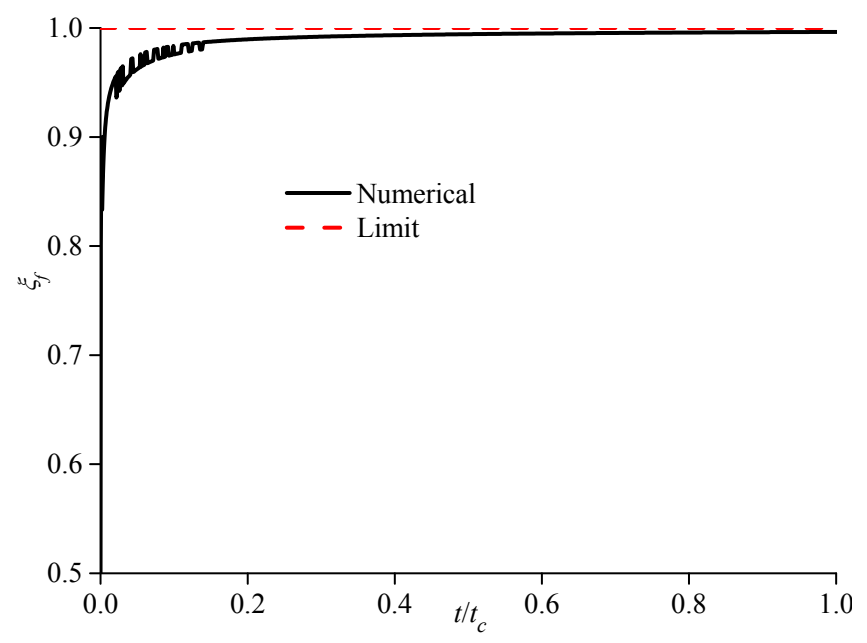


Fig. 8. Evolution of $\xi_{f}$ in the simulation.

Fig. 9 shows $e_{p(0)}, e_{w(0)}$, the normalized net pressure profiles, and fracture with profiles when $l$ reaches $13.5 \mathrm{~m}$. In the numerical result $l$ reaches $l_{e}$ when $t$ reaches 15.08s. It is seen in Fig. 9(a) and (b) that the relative errors generally decrease when the fracture propagates. This means that the numerical solution gets closer to the self-similar solution when the fracture propagates. The net pressure profile (see Fig. 9(c)) and the fracture width profile (see Fig. 9(d)) show that the self-similar solution can be well approximated by the numerical results when $l$ reaches $l_{e}$.

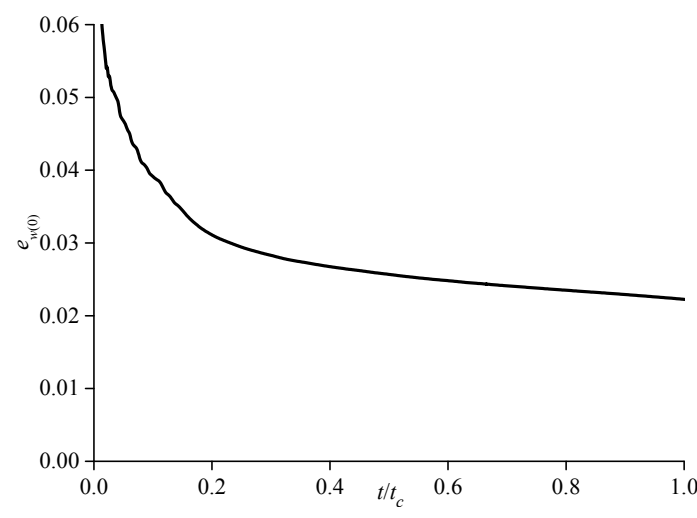

(a)

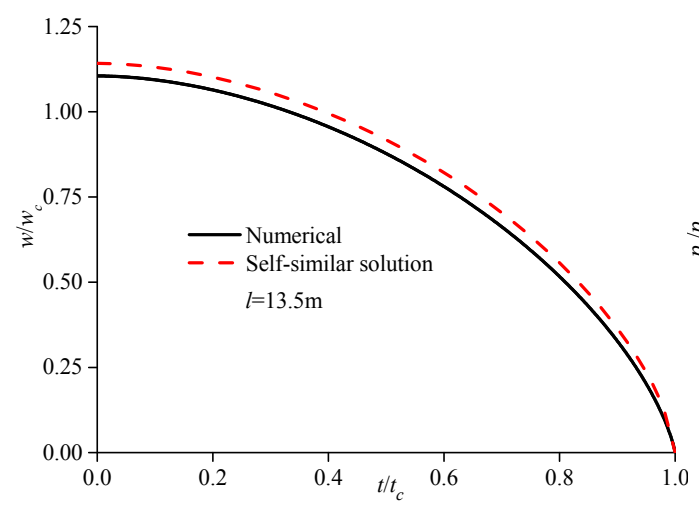

(c)

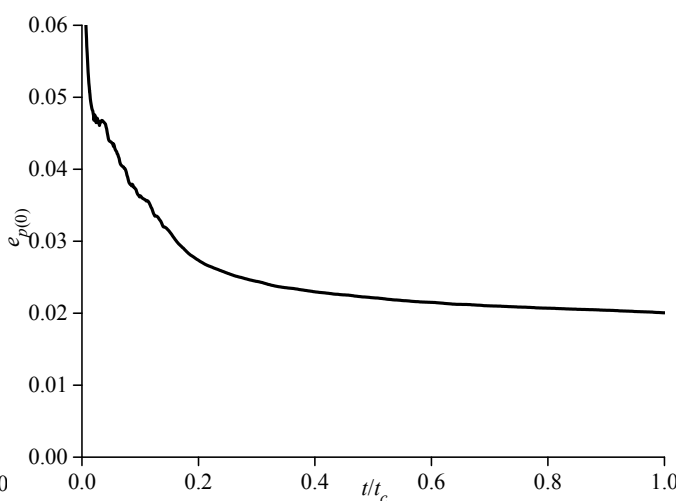

(b)

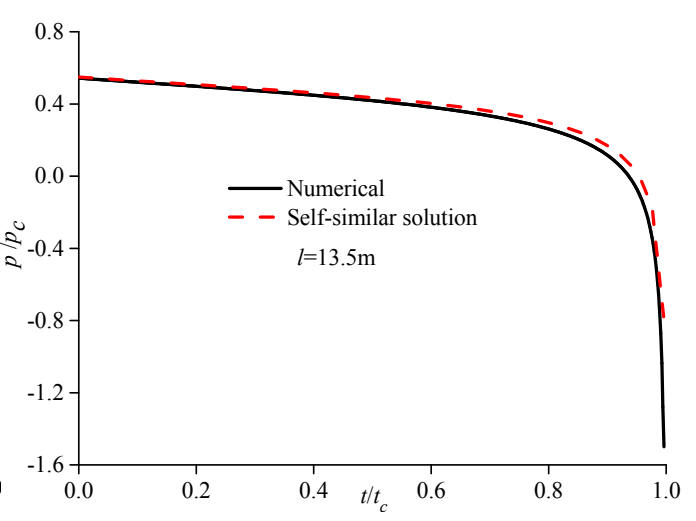

(d)

Fig. 9. $e_{p(0)}(\mathrm{a}), e_{w(0)}(\mathrm{b})$, the normalized pressure profile (c), and the normalized fracture width profile with when $l=13.5 \mathrm{~m}(\mathrm{~d})$. 
We presented three examples to simulate hydraulic fracturing with constant fluid lag fraction, without fluid lag, and with vanishing fluid lag. The numerical results in these examples have excellent agreement with the self-similar analytical solutions. This excellent agreement verifies the unified finite element method. Some suction zone is allowed to exist temporally in our numerical method. In the self-similar solutions without fluid lag, suction zone always exists as the net pressure at the fracture tip is negative and infinite. However, there is no suction zone in the self-similar analytical solution with a fluid lag. In the semi-analytical solutions the fracture tip is always at its critical state. In our method the stress intensity factor is always smaller than the fracture toughness when a fluid lag exists, while larger than the fracture toughness when there is no fluid lag. The differences in these two aspects contribute in part to their simulation differences shown in Figs. 6, 7 and 9.

We define suction zone ratio as the ratio of suction zone length to fluid length. The evolution of suction zone ratio in the simulation where $K_{m}$ equals 0.8 and $\sigma_{0}$ equals 0 is shown in Fig. 9 for the first 100 steps, as well as the ratio of $K_{I}$ to $K_{I C}$. It is seen in Fig. 9(a) that the suction zone is always a very small one, and it tends to disappear once it occurs. This is a general trend in all the fluid lag simulations. It is seen in Fig. 9(b) that only on the very beginning the stress intensity factor is far smaller than the fracture toughness, later it is very close to the fracture toughness. Although Eq. (7) is not satisfied in our method, it is well approximated in our method in a non-mandatory way. This means that our suction zone assumption and physically-acceptable scheme are reasonable and reliable. This is reflected by the satisfactory agreement between the numerical simulations and the analytical solutions. 


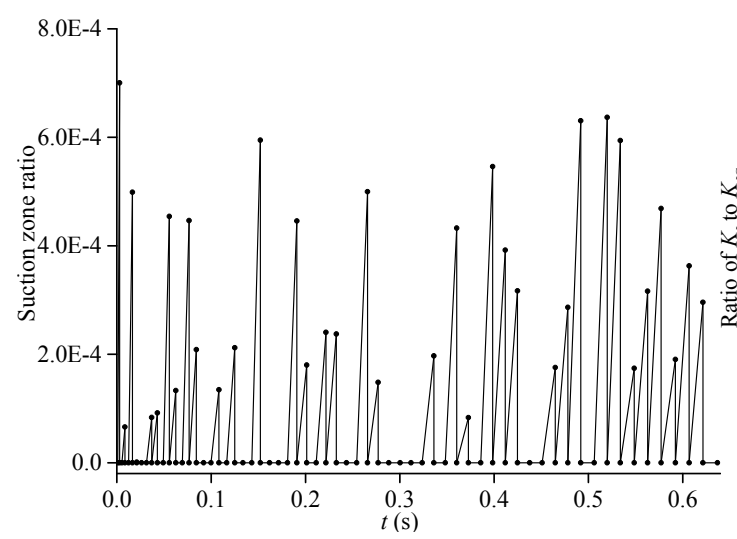

(a)

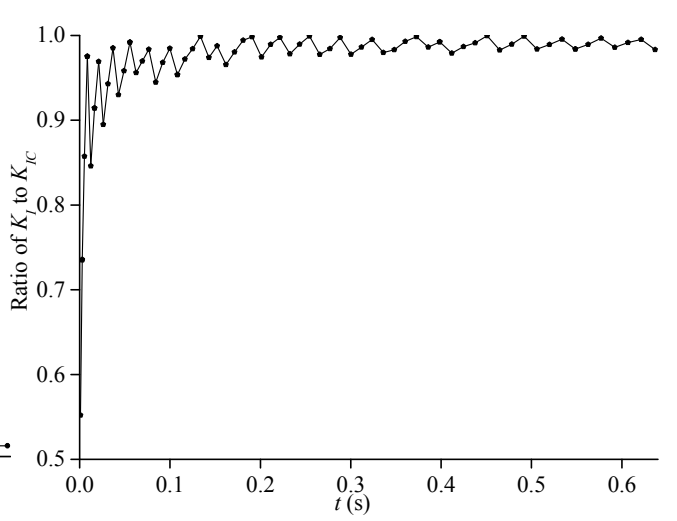

(b)

Fig. 10. Evolutions of suction zone ratio (a) and the ratio of $K_{I}$ to $K_{I C}(b)$ in the first 100 steps of the simulation with $K_{m}=0.8$ and $\sigma_{0}=0$.

We discuss the propagation of a fracture with long initial length based on the unified method. The example with $K_{m}$ equal to 2 and $\sigma_{0}$ equal to 0 in the verification section is used. The initial half length of the fracture equals $7.5 \mathrm{~m}$. The initial half fluid length and the initial pressure are the same as those in the verification section. It is found in the simulation that the fracture is completely filled by the fluid at the moment of $6.44 \mathrm{~s}$, and it begins to propagate at the moment of 7.40s. Fig. 11 shows $p(0), w(0)$, and the pressure and fracture profiles of the fracture at some moments. Just as shown in Fig. 11, p(0) decreases before the gap vanishes, and increases for a while when the fracture is completely filled by the fluid, and then decreases again after some fracture propagation. Similar phenomenon is observed in [30]. However, w(0) always increases. It is also seen in Fig. 11 that fracture width and pressure profiles before fracture propagation have different patterns from those after fracture propagation. 


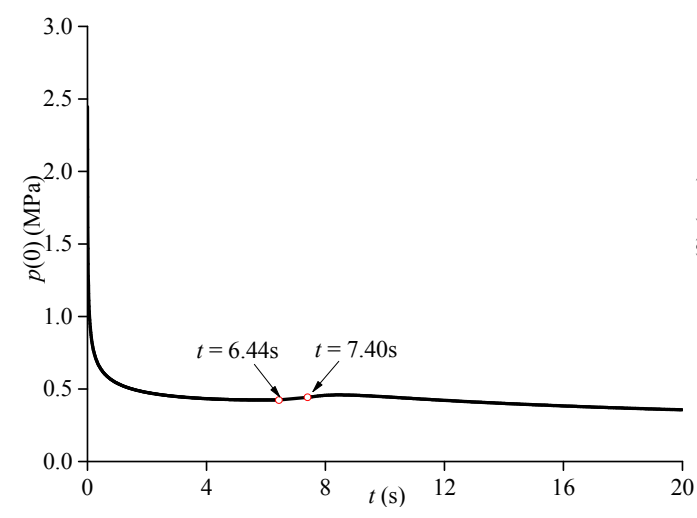

(a)

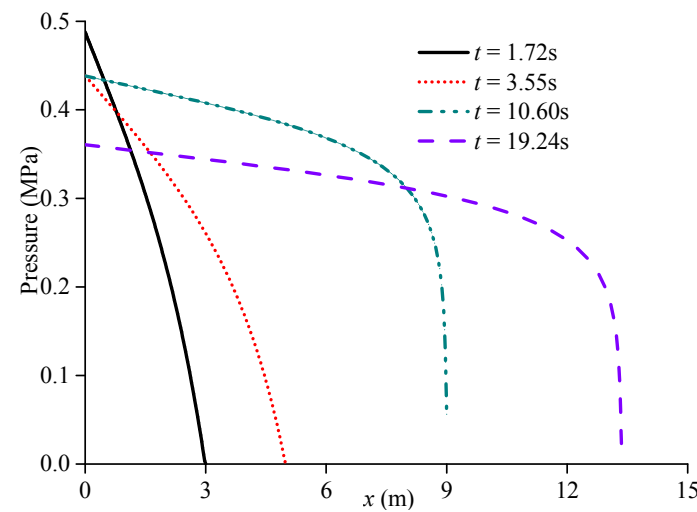

(c)

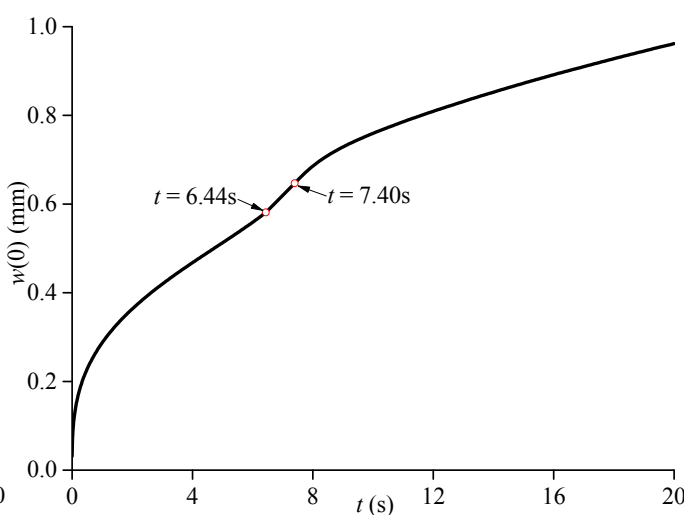

(b)

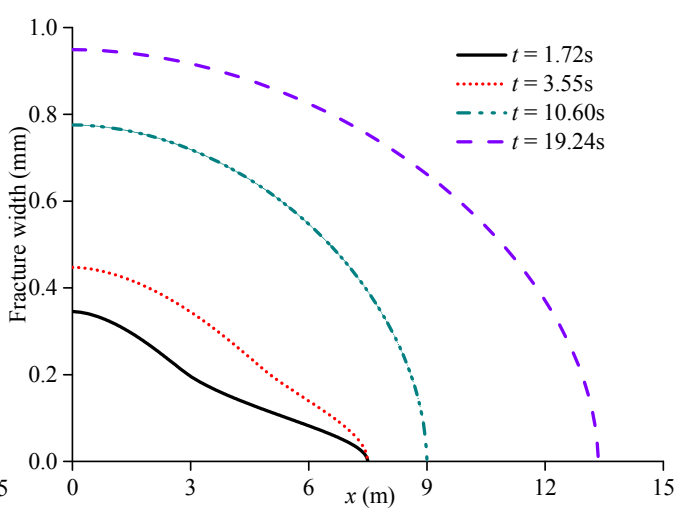

(d)

Fig. 11. $p(0)(\mathrm{a}), w(0)(\mathrm{b})$, pressure profiles $(\mathrm{c})$, and fracture width profiles $(\mathrm{d})$.

It is seen in Eqs. (25-26) that simulations are controlled by $\varepsilon_{p}^{l}$ and $\varepsilon_{p}^{u}$ when a fluid lag exists. We re-run the examples where $K_{m} \mathrm{~s}$ are smaller than 1 and $\sigma_{0} \mathrm{~s}$ equal 0 with another two sets of $\varepsilon_{p}^{l}$ and $\varepsilon_{p}^{u} \cdot e_{w(0)} \mathbf{s}$ in the simulations are plotted in Fig. 12 when $l$ reaches $l_{c}$. It is seen in Fig. 12 that $e_{w(0)} \mathrm{s}$ do not have large variation except the simulation with $\gamma_{m}$ equal to 0.5 , and $e_{w(0)} \mathrm{s}$ are smaller than 0.012 even when the magnitudes of $\varepsilon_{p}^{l}$ and $\varepsilon_{p}^{u}$ equal 0.01 . The unified method is not sensitive to $\varepsilon_{p}^{l}$ or $\varepsilon_{p}^{u}$. 


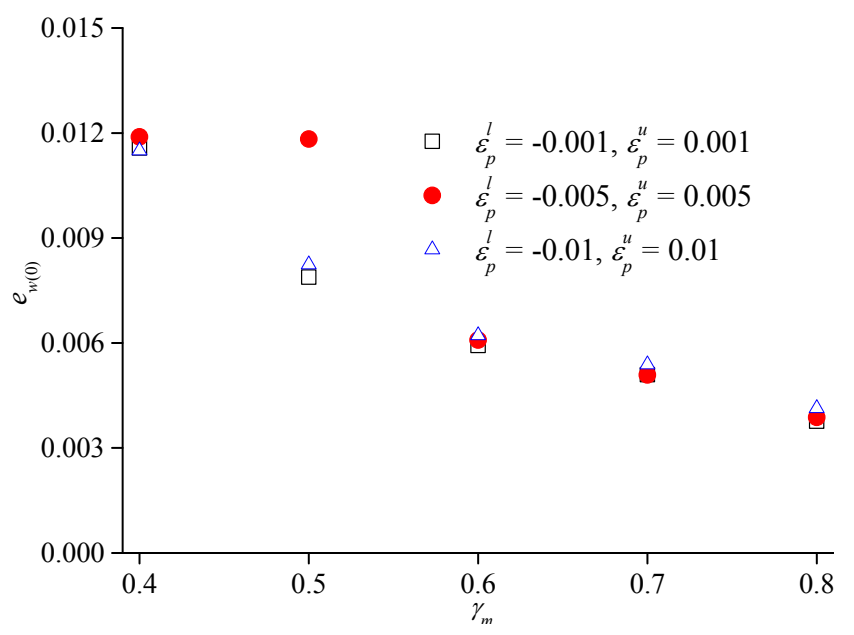

Fig. 12. $e_{w(0)} \mathrm{s}$ in the simulations where $K_{m} \mathrm{~s}<1$ and $\sigma_{0} \mathrm{~s}=0$.

All the simulations were run by an in-house program. No numerical instability is observed in the simulations, and mass conservation of the injected fluid is guaranteed. Iteration numbers of solving the coupled Eqs. (20) and (21) in some of the simulations in the verification section are plotted in Fig. 13 for their first 100 steps. It is seen in Fig. 11 that in most steps the solutions get convergent within 5 iterations. This summary is applicable to all other simulations. This means that the numerical method has acceptable convergence.

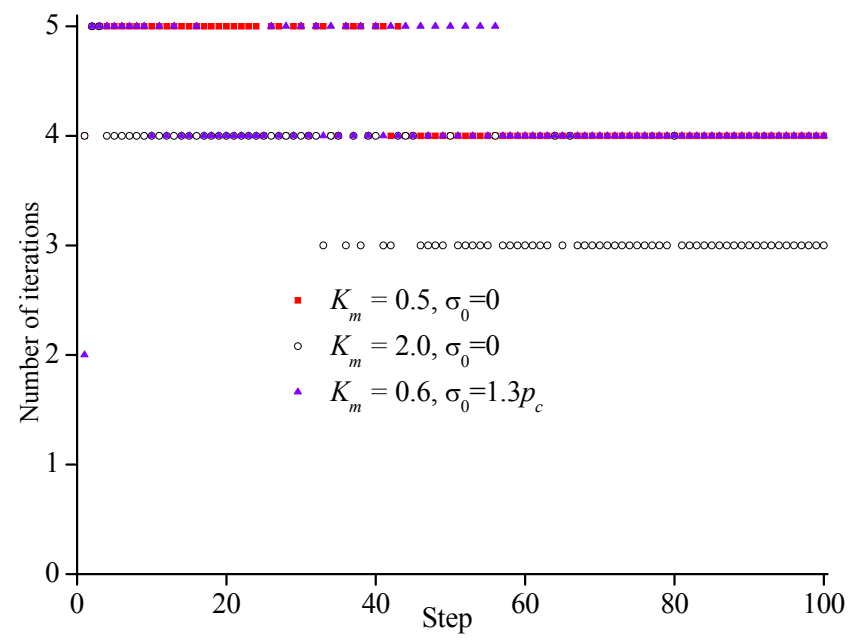




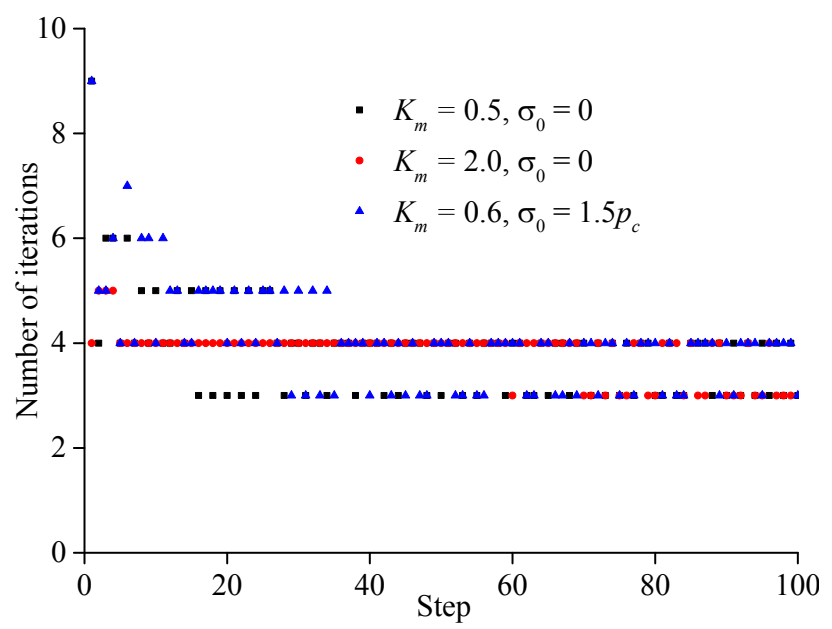

Fig. 13. Iteration numbers of some simulations in their first 100 steps.

\section{Conclusion}

In this paper we proposed a unified finite element method to simulate hydraulic fracturing with and without fluid lag. This method is proposed based on the basis of a unified boundary condition on the fluid front, where a suction zone is allowed to exist temporarily. In the unified method a physically-acceptable scheme is used, where no effort is needed to track fluid front explicitly, and the burden of model re-meshing induced by fluid front advancement is also avoided. We used the unified method to simulate hydraulic fracturing with a constant fluid lag ratio, without fluid lag, and with a vanishing fluid lag. The method is completely verified by comparing its simulations with analytical solutions. Simulations show that our assumption and scheme are reasonable and reliable. The unified method shows excellent stability and convergence.

\section{Acknowledgements}

Funding for this project is provided by RPSEA through the "Ultra-Deepwater and Unconventional Natural Gas and Other Petroleum Resources" program authorized by the U.S. Energy Policy Act of 2005. RPSEA (www.rpsea.org) is a nonprofit corporation whose mission is to provide a stewardship role in ensuring the focused research, development and deployment of safe and environmentally responsible 
technology that can effectively deliver hydrocarbons from domestic resources to the citizens of the United States. RPSEA, operating as a consortium of premier U.S. energy research universities, industry, and independent research organizations, manages the program under a contract with the U.S. Department of Energy's National Energy Technology Laboratory. We appreciate the anonymous reviewers' great effort for the improvement of the article.

\section{Reference}

1. Bao, J.Q., E. Fathi, and S. Ameri, Uniform investigation of hydraulic fracturing propagation regimes in the plane strain model. International Journal for Numerical and Analytical Methods in Geomechanics, 2015. 39: p. 507-523.

2. Adachi, J., et al., Computer simulation of hydraulic fractures. International Journal of Rock Mechanics and Mining Sciences, 2007. 44(5): p. 739-757.

3. Lister, J.R., Buoyancy-driven fluid fracture: the effects of material toughness and of low-viscosity precursors. Journal of Fluid Mechanics, 1990. 210: p. 263-280.

4. Spence, D.A. and D.L. Turcotte, Magma-driven propagation of cracks. Journal of Geophysical Research, 1985. 90: p. 575-580.

5. Tsai, V.C. and J.R. Rice, A model for turbulent hydraulic fracture and application to crack propagation at glacier beds. Journal of Geophysical Research, 2010. 115(F3): p. 227-235.

6. Murdoch, L.C., Mechanical analysis of ideaized shallow hydraulic fracture. Journal of Geotechnical and Geoenvironmental Engineering, 2002. 128(6): p. 488-495.

7. Jeffrey, R.G. and K.W. Mills, Hydraulic fracturing applied to inducing longwall coal mine goaffalls, in Pacific Rocks 2000. 2000, Balkema: Rotterdam. p. 423-430.

8. Barenblatt, G.I., On the formation of horizontal cracks in hydraulic fracture of an oil-bearing stratum, Prikl. Mechanics of Materials, 1956. 20: p. 475-486.

9. Geertsma, J. and F.D. Klerk, A rapid method of predicting width and extent of hydraulic induced fractures. Journal of Petroleum Technology, 1969. 21(12): p. 1571-1581.

10. Khristianovic, S.A. and Y.P. Zheltov, Formation of vertical fractures by means of highly viscous liquid, in Proceedings of the Fourth World Petroleum Congress. 1955: Rome. p. 579-586.

11. Lecampion, B. and E. Detournay, An implicit algorithm for the propagation of a hydraulic fracture with a fluid lag. Computer Methods in Applied Mechanics and Engineering, 2007. 196: p. 4863-4880.

12. Murdoch, L.C., Hydraulic fracturing of soil during laboratory experiments: methods and observations. Geotechnique, 1993. 43(2): p. 255-265.

13. Economides, M.J. and K.G. Nolte, Reservoir Stimulation (Third Edition). 2000, New York: John Wiley \& Sons, Ltd.

14. Zhang, X., R.G. Jeffrey, and E. Detournay, Propagation of a hydraulic fracture parallel to a free surface. International Journal for Numerical and Analytical Methods in Geomechanics, 2005. 29(13): p. 1317-1340. 
15. Garagash, D., Propagation of a plane-strain hydraulic fracture with a fluid lag: Early-time solution. International Journal of Solids and Structures, 2006. 43(18-19): p. 5811-5835.

16. Detournay, E., Propagation regimes of fluid-driven fractures in impermeable rocks International Journal of Geomechanics, 2004. 4(1): p. 35-45.

17. Garagash, D.I., Plain-strain propagation of a fluid-driven fracture during injection and shut-in: Asymptotics of large toughness. Engineering Fracture Mechanics, 2006. 73(4): p. 456-481.

18. Adachi, J. and E. Detournay, Self-similar solution of a plane-strain fracture driven by a power-law fluid. International Journal for Numerical and Analytical Methods in Geomechanics, 2002. 26: p. 579-604.

19. Adachi, J. and E. Detournay, Plane strain propagation of a hydraulic fracture in a permeable rock. Engineering Fracture Mechanics, 2008. 75(16): p. 4666-4694.

20. Garagash, D. and E. Detournay, Plane-strain propagation of a fluid-driven fracture: small toughness solution. Journal of Applied Mechanics, 2005. 72(6): p. 916-928.

21. Spence, D.A. and P.W. Sharp, Self-similar solution for elastohydrodynamic cavity flow. Proceedings of the Royal Society A: Mathematical, Physical and Engineering Sciences, 1985. 400(1819): p. 289-313.

22. $\mathrm{Hu}, \mathrm{J}$. and D.I. Garagash, Plane-strain propagation of a fluid-driven crack in a permeable rock with fracture toughness. ASCE Journal of Engineering Mechanics, 2010. 136(9): p. 1152-1166.

23. Chen, Z.R., et al., Cohesive zone finite element-based modeling of hydraulic fracturing. Acta Mechanica Solid Sinica, 2009. 22(5): p. 443-452.

24. Chen, Z., Finite element modelling of viscosity-dominated hydraulic fractures. Journal of Petroleum Science and Engineering, 2012. s 88-89(2): p. 136-144.

25. Carrier, B. and S. Granet, Numerical modeling of hydraulic fracture problem in permeable medium using cohesive zone model. Engineering Fracture Mechanics, 2012. 79: p. 312-328.

26. Bao, J.Q., E. Fathi, and S. Ameri, A coupled finite element method for the numerical simulation of hydraulic fracturing with a condensation technique. Engineering Fracture Mechanics, 2014. 131(2): p. 269-281.

27. Gordeliy, E. and E. Detournay, A fixed grid algorithm for simulating the propagation of a shallow hydraulic fracture with a fluid lag. International Journal for Numerical and Analytical Methods in Geomechanics, 2011. 35(5): p. 602-629.

28. Hunsweck, M.J., Y.X. Shen, and A.J. Lew, A finite element approach to the simulation of hydraulic fractures with lag. International Journal for Numerical and Analytical Methods in Geomechanics, 2013. 37(9): p. 993-1015.

29. Gordeliy, E. and A. Peirce, Coupling schemes for modeling hydraulic fracture propagation using the XFEM. Computer Methods in Applied Mechanics and Engineering, 2013. 253(1): p. 305-322.

30. Shen, Y., A variational inequality formulation to incorporate the fluid lag in fluid-driven fracture propagation. Computer Methods in Applied Mechanics and Engineering, 2014. 272(2): p. 17-33.

31. Fu, P., S.M. Johnson, and C.R. Carrigan, An explicitly coupled hydro-geomechanical model for simulating hydraulic fracturing in arbitrary discrete fracture networks. International Journal for Numerical and Analytical Methods in Geomechanics, 2012. 37: p. 2278-2300. 
32. Gupta, P. and C.A. Duarte, Simulation of non-planar three-dimensional hydraulic fracture propagation. International Journal for Numerical and Analytical Methods in Geomechanics, 2014. 38: p. 1397-1430.

33. Batchelor, G.K., An introduction to fluid dynamics. 1967, Cambridge, UK: Cambridge University Press.

34. Boone, T.J. and A.R. Ingraffea, A numerical procedure for simulation of hydraulically-driven fracture propagation in poroelastic media. International Journal for Numerical and Analytical Methods in Geomechanics, 1990. 14(1): p. 27-47.

35. Devloo, P.R.B., et al., A finite element model for three dimensional hydraulic fracturing. Mathematics and Computers in Simulation, 2006. 73(s 1-4): p. 142-155. 